\title{
THE INFLUENCE OF CROWDING ON DENIS POINT SOURCE DETECTION RATES
}

\author{
ANDRÁS HOLL* \\ Konkoly Observatory, P.O. Box 67, H-1525, Budapest, Hungary \\ and \\ ERIK DEUL \\ Sterrewacht Leiden, P.O. Box 9513, 2300 RA Leiden, The Netherlands
}

\begin{abstract}
In this paper we estimate the effects of crowding (resulting in failure to detect sources due to unresolvable blends) on DENIS point source extraction. Crowding, together with confusion (flux contribution from sources below the detection limit), is expected to be a major quality-degrading factor close to the galactic plane. Correcting for the incompleteness of the DENIS data could produce accurate starcounts very close to the galactic plane.
\end{abstract}

Key words: DENIS - source extraction - crowding

\section{Introduction}

Averaged over the entire celestial hemisphere, about 100 sources $\left(\mathrm{m}_{\mathrm{K}}<\right.$ 14.0) are expected per DENIS frame; close to the galactic plane, however, this number will be of the order of thousands or tens of thousands. Based on the observations of Davidge (1991) in the direction of the Baade Window at $b=-4^{\circ}$ we might expect about 5000 stars in a $\mathrm{K}$ band frame. To describe the density and the brightness distribution of stars we used models of Robin (see the paper of Robin in this volume, and Robin \& Crézé, 1986). Applying Monte-Carlo methods to assess the crowding loss, we first created artificial frames using IRAF and then tried to retrieve the input objects. For source extraction the standard DENIS source extraction code was used (Deul 1993).

\section{Method}

The frames were simulated with a stellar brightness distribution given by the model for $l=0^{\circ}$ and $b=6^{\circ}$; stars were added to the test frames up to one magnitude fainter than the limiting magnitude (the expected limiting magnitudes are 14.0, 16.0 and 18.0 in $\mathrm{K}^{\prime}$, J and I, respectively). The total number of stars added to the frame were 25000, 9100, and 4300 . However, only those above the nominal limiting magnitude were used in the computations; namely 3177 in $\mathrm{K}^{\prime}, 6130$ in $\mathrm{J}$ and 14569 in $\mathrm{I}$; the fainter

* Currently at Sterrewacht Leiden 
stars were added to contribute to the noise. The spatial distribution of the test stars was Poissonian. Some non-blended stars close to the detection limit were lost due to noise, but we have corrected our results for this effect, using derived detection probabilities for isolated stars in test frames consisting of stars distributed with small random displacements around a grid. The noise parameters used for the creation of the test images are based on expected detector characteristics. All the test images were made using the artificial data package of IRAF.

The test images were fed to the standard DENIS source extraction code. The parameters of the extraction code were tuned using artificial frames. Final tuning, however, is possible only with real data, so the parameters which will be used during the actual survey will certainly differ.

The sources found by the extraction code were associated with the input objects, applying a wide window constraint in coordinates, using an image with sufficient number of objects to provide fair statistics, but not so crowded as to lead to a large number of false associations. The derived photometric parameter was converted to magnitudes, and the standard deviations of positional and photometric errors were computed.

To decrease the number of false associations the association program was run once more with $3 \sigma$ constraints imposed on both position and photometry. Thus a few objects with large positional errors (some of the bright stars, for instance) were missed. Multiple associations with the same input object were then excluded, and finally, the detection rate and object-toobject distance statistics were computed. For the detection rate calculation the magnitude values of the associated input objects were used. With this method we have avoided the 'inflation' of source counts in a given magnitude bin (the errors in the photometry shift objects to neighbouring bins, and there are more objects in the fainter bins than in the brighter ones). The detection-rate values for bright stars are unreliable due to the poor number statistics. They may perhaps have large photometric and/or positional errors caused by the faint stars that are unresolvably blended to their image.

\section{Conclusion}

In spite of the high object density (i.e. high number of objects per frame), a large fraction of the stars can still be detected: only $15 \%\left(\mathrm{~K}^{\prime}\right), 25 \%(\mathrm{~J})$, and $21 \%$ (I) were lost. For 2000 stars images the crowding loss is only about $8 \%$ (in all three bands, assuming brightness distributions like that of the direction of $l=0^{\circ}, b=6^{\circ}$. In case of 1 " seeing the sources further apart than $4.5^{\prime \prime}\left(\mathrm{K}^{\prime}\right.$ and J) or 3" (I) will certainly be deblended; for smaller separations the detection probability drops rapidly (Fig. 1.a). Using the computed detection rates as a function of magnitude (Fig. 1.b-d) one can correct for the incompleteness caused by crowding. 

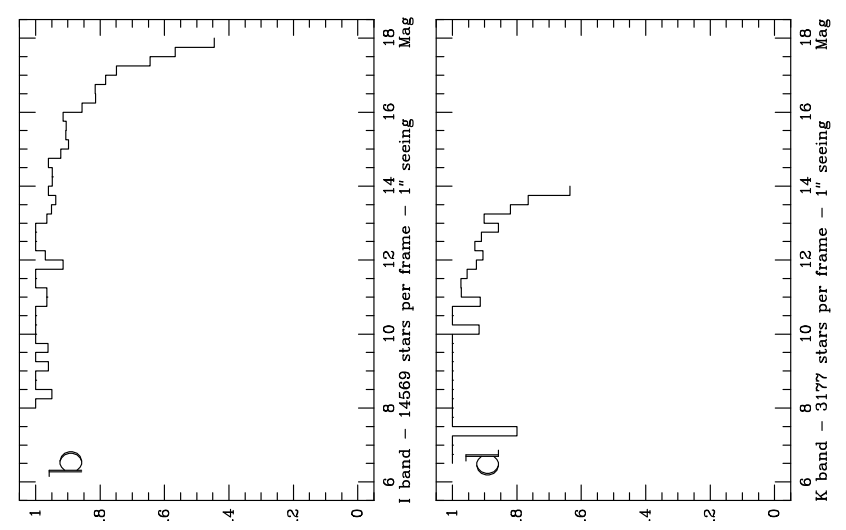

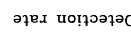

әุв. иоเฺฺวәาәด
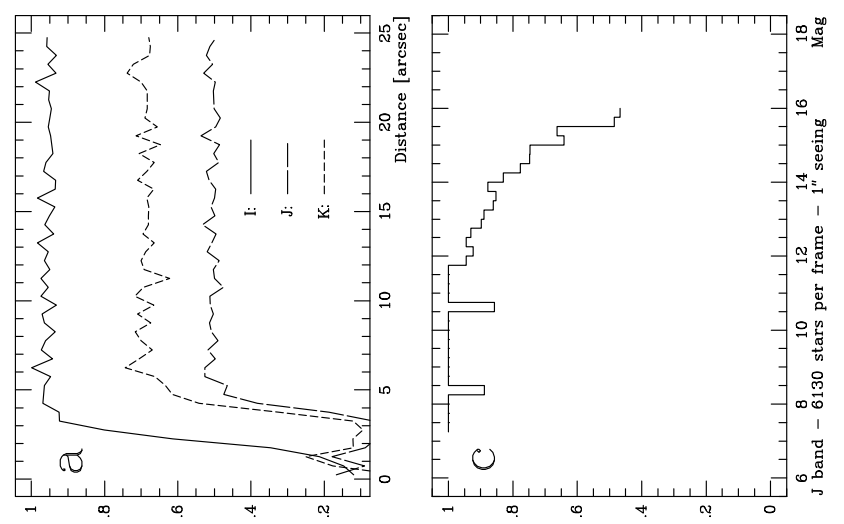

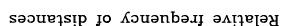

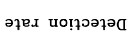

Fig. 1. a: The ratio of the frequency of a given separation in the input coordinate list to that of the detected objects. b-d: Detection rates for the I, J, and $\mathrm{K}^{\prime}$ bands; the only effect taken into account is the crowding

\section{Acknowledgements}

A. Holl is grateful for the support from the EEC "Cooperation in Science and Technology with Central and Eastern European Countries" grant (proposal No. 2905) and the generous help of the organizers of the Les Houches conference; E. Deul and A. Holl acknowledge the use of the NATO Research grant No. 928006. 


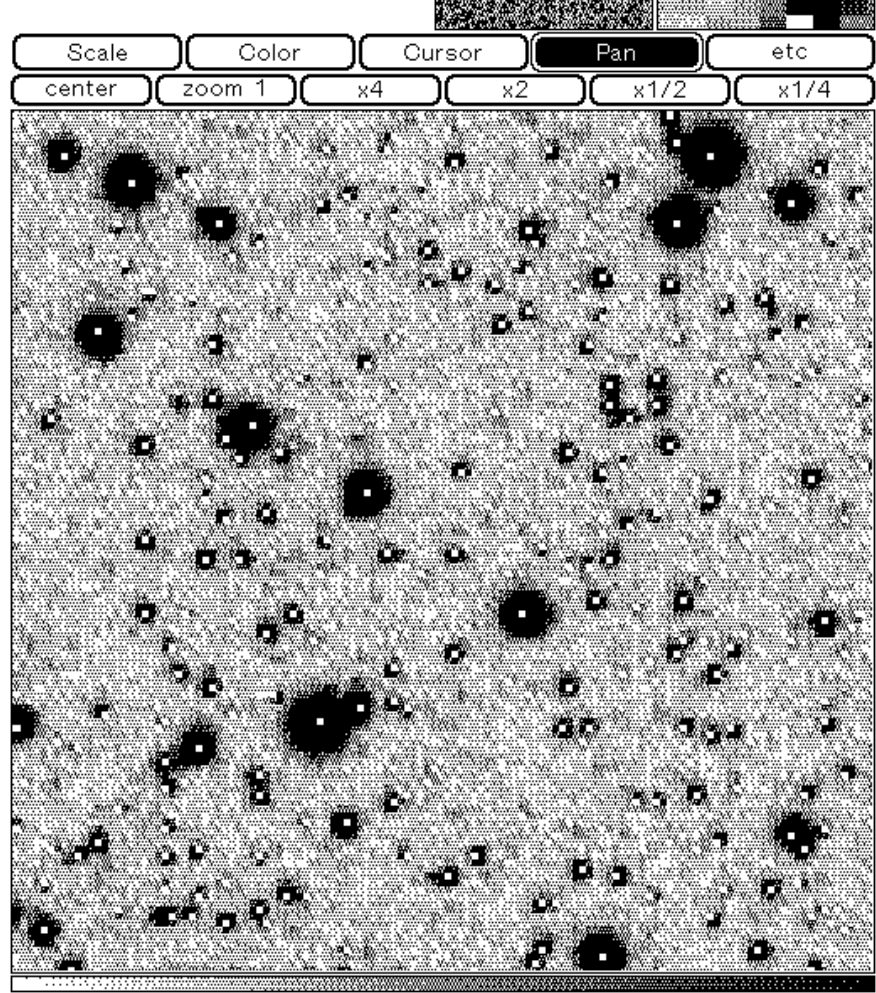

Fig. 2. Part of the I band image, the detections are marked with white dots

\section{References}

Robin, A.C.: 1993, 'Synthesis of galactic stellar populations and expected sources in infrared surveys' in: Science with astronomical near-infrared surveys (this volume)

Robin, A.C. and Crézé, M.: 1986, 'Stellar populations in the Milky Way: a synthetic model' Astronomy and Astrophysics vol. 157, 71

Davidge, T.J.: 1991, 'JHK imaging of a field in Baade's Window' Astrophysicsl Journal vol. 380, 116

Deul, E.: 1993, 'Source extraction in the DENIS survey' in: Proceedings of the 5th ESOST/ECF Data Analysis Workshop, Garching (in press) 